\title{
Vacuum ultraviolet circularly polarized coherent femtosecond pulses from laser seeded relativistic electrons
}

\author{
N. Čutić, F. Lindau, S. Thorin, and S. Werin \\ MAX-lab, Lund University, P.O. Box 118, S-221 00 Lund, Sweden \\ J. Bahrdt, W. Eberhardt, and K. Holldack \\ Helmholtz-Zentrum Berlin für Materialien und Energie GmbH, Wilhelm-Conrad-Röntgen-Campus, \\ Albert-Einstein-Straße 15, D-12489 Berlin, Germany \\ C. Erny, A. L'Huillier, and E. Mansten \\ Physics Department, Lund University, P.O. Box 118, S-221 00 Lund, Sweden
}

(Received 9 December 2010; published 28 March 2011)

\begin{abstract}
We have demonstrated the generation of circularly polarized coherent light pulses at $66 \mathrm{~nm}$ wavelength by combining laser seeding at $263 \mathrm{~nm}$ of a $375 \mathrm{MeV}$ relativistic electron bunch with subsequent coherent harmonic generation from an elliptical undulator of APPLE-II type. Coherent pulses at higher harmonics in linear polarization have been produced and recorded up to the sixth order $(44 \mathrm{~nm})$. The duration of the generated pulses depends on the temporal overlap of the initial seed laser pulse and the electron bunch and was on the order of $200 \mathrm{fs}$. Currently, this setup is the only source worldwide producing coherent fs-light pulses with variable polarization in the vacuum ultraviolet.
\end{abstract}

DOI: 10.1103/PhysRevSTAB.14.030706

PACS numbers: 41.60.Cr, 42.65.Ky, 42.25.Ja

\section{INTRODUCTION}

Brilliant synchrotron radiation as produced in third generation storage rings is used in many user facilities worldwide featuring polarization control of the radiation using elliptical undulators. However, in the vacuum ultraviolet (VUV) and x-ray regime the synchrotron radiation is only partially coherent transversely, and multiparticle (gain) effects are nonexistent. Time resolved measurements are limited by the electron bunch length of typically 10-100 ps. Specific operation conditions, so-called low-alpha optics, provide bunch lengths on the ps scale [1]. The pulse duration can be further reduced with slicing techniques, where only a part of the bunch charge is used $[2,3]$. However, these pulses are temporally incoherent and the photon flux is limited.

Coherence properties as well as the photon flux can be significantly improved with free-electron lasers (FELs). Self-amplified spontaneous emission (SASE) FELs generate high peak brilliance, transverse coherence, short pulse lengths, and wavelengths [4-6] but since they start from noise they suffer from intensity variations, the signal longitudinally coherent only within the slippage length (shorter than the bunch length), and spiky resulting spectra. Introduction of a seed pulse (to avoid buildup from noise) significantly improves longitudinal coherence but creates

Published by American Physical Society under the terms of the Creative Commons Attribution 3.0 License. Further distribution of this work must maintain attribution to the author(s) and the published article's title, journal citation, and DOI. new challenges to find a suitable seed source. Various seeding schemes have been proposed or carried out: seeding with high harmonics generated in a gas $[7,8]$, self-seeding schemes [9], extraction of a harmonic after bunching at a long wavelength [high-gain harmonic generation (HGHG)] [10-13], or recently by echo-enabled harmonic generation [14].

The radiation from these sources [4-14] is linearly polarized since planar radiators were used. In the VUV regime, quarter wave plates converting linear to circular light are not available. One has to rely on reflection optics with low reflection efficiency and small tuning ranges. Thus, the linearly polarized radiation of existing FELs or the radiation from a high harmonic generation source cannot be easily converted to circular polarization using optics and helical undulators have to be used instead. The FERMI project which is under construction will have variably polarizing radiators [15].

Here, we show the first experimental results of combining temporal coherence, circular polarization, and fs pulse length down to $66 \mathrm{~nm}$ wavelength using the experimental layout depicted in Fig. 1. The same layout is also used to generate even higher harmonics in linear polarization (down to $44 \mathrm{~nm}$ ).

The method we used is similar to high-gain harmonic generation (HGHG) as in Ref. [11] but with the differences that we use an APPLE-II type undulator as a radiator to produce harmonics with variable polarization, and our seed pulse is in the UV region. The modulator undulator (although tunable) is set to resonance with the seed laser frequency $\omega$, whereas the radiator undulator fundamental 


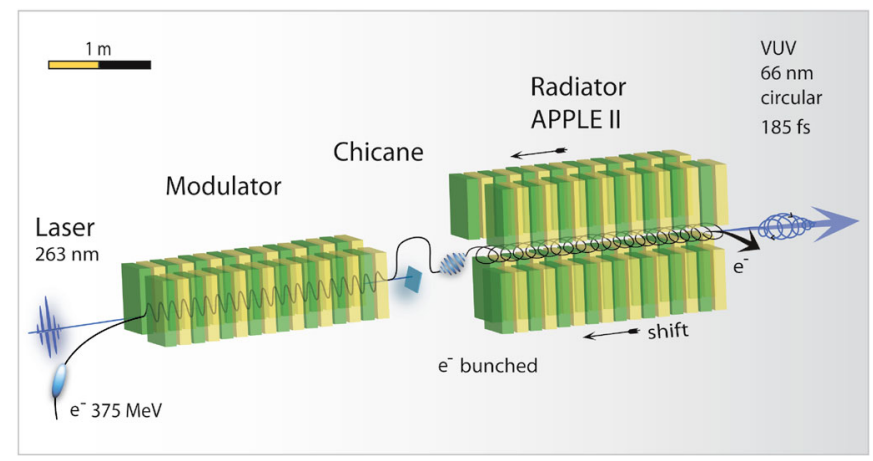

FIG. 1. Principle of the experimental setup of the MAX-lab test FEL (undulator section). Seeding occurs at $263 \mathrm{~nm}$ in the modulator which applies a horizontal field to the electron beam. After bunching in a dispersive section (chicane), the radiator emits circularly polarized coherent pulses up to the 4th harmonic at $66 \mathrm{~nm}$.

mode is set to $n \omega(n \geq 1)$. Since the length of our radiator is such that the exponential amplification process just begins, we refer to this method as coherent harmonic generation (CHG) instead of HGHG.

\section{EXPERIMENTAL SETUP AND METHODS}

The complete experimental setup is shown in Fig. 2. The four major parts of our setup (the accelerator, the laser system, the undulator section, and the diagnostics) are presented in this section.

\section{A. The accelerator}

The test FEL facility is built around the MAX injector [16] which in normal operation provides the injection beam for the three storage rings at MAX-lab (MAX I, II, and III). The electron source is an rf gun [17] which can be operated both in thermal mode (ring injection) and in photocathode mode (test FEL)[18]. The electron bunches for the test FEL are created by a Ti:sapphire gun laser system. The pulse length is $10 \mathrm{ps}$. The rf accelerating phase in the gun is adjusted relative to the laser pulse to achieve minimum emittance. The main acceleration is done in two $3 \mathrm{GHz}$ linac structures. The electron beam is recirculated such that the linacs are passed twice and a total energy of $375 \mathrm{MeV}$ is reached. The beam exits the recirculator

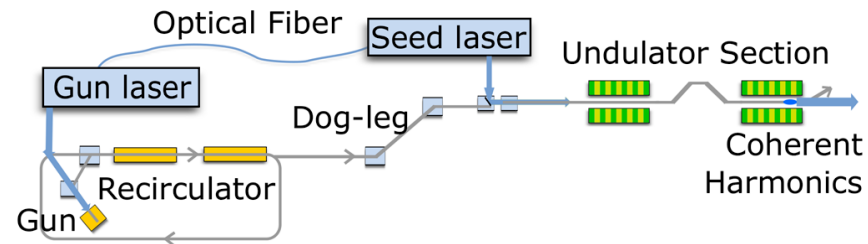

FIG. 2. Layout of the CHG-FEL test setup at MAX-lab, including electron accelerator, undulator section, the seed laser beam line, as well as the laser synchronization scheme. through a chicane and is transported from basement level to ground level by a dog-leg. These latter systems provide the necessary $\mathrm{R}_{56}$ (momentum compaction) to compress the electron bunches.

After longitudinal compression the beam enters the test FEL beam line via a "half chicane" which lifts the beam $20 \mathrm{~mm}$. This allows us to inject the seed laser beam on top of the electron bunch. A slight remaining dispersion is created by the half chicane, but the effect is negligible compared to the electron beam size. Focusing is achieved by a quadrupole doublet in the transport line upstream of the half chicane.

\section{B. The laser system}

The laser system, providing pulses for photocathode gun and seeding, is locked to the master rf oscillator by locking the master laser oscillator (Femtolasers Synergy, $93.71 \mathrm{MHz}, 790 \mathrm{~nm}$ central wavelength, $13 \mathrm{~nm}$ FWHM bandwidth) with a jitter of about $0.2 \mathrm{ps}$. The laser oscillator pulses are stretched and split into two branches: one branch for the gun laser where it is subsequently amplified and tripled $(263 \mathrm{~nm}, 10 \mathrm{ps}, 500 \mu \mathrm{J})$ to be used for the photocathode gun; the second branch of the oscillator pulse is guided through a $90 \mathrm{~m}$ polarization maintaining fiber to the seed laser where it is also amplified, compressed, tripled, delayed (to regulate arrival time), and focused into the modulator undulator $(263 \mathrm{~nm}, 500 \mathrm{fs}$, up to $100 \mu \mathrm{J})$. A small part of the infrared seed pulse before the tripling is sampled to be used for an electro-optical detection.

\section{The undulator section}

The undulator section consists of two undulators and a dispersive section. In the first undulator, which is called the modulator, the electrons interact with a $263 \mathrm{~nm}$ photon beam from the Ti:sapphire laser. For the current experiments it is operated at $10 \mu \mathrm{J}$ pulse energy (maximum $100 \mu \mathrm{J}$ ) and $0.5 \mathrm{ps}$ pulse length. The modulator must be resonant with the seed photon energy to imprint an energy modulation to the electron bunch. Within a dispersive section the energy modulation is subsequently converted into a spatial modulation. The required energy modulation $\Delta \gamma$ is determined by the natural relative energy spread $\sigma_{E}$. Beam bunching efficient enough for the $\mathrm{CHG}$ at a certain harmonic $n$ requires $\Delta \gamma \geq \sigma_{E} \cdot n$, e.g., an energy spread $\sigma_{E}=5 \times 10^{-4}$ requires an energy modulation of $\Delta \gamma=$ $1.5 \times 10^{-3}$ and $2.5 \times 10^{-3}$ for the 3rd and 5th bunching harmonic, respectively.

The modulator is a pure permanent magnet planar device with a period length of $48 \mathrm{~mm}$ and 30 periods. At a minimum gap of $13.2 \mathrm{~mm}$ a $K$ parameter of 3.52 is achieved. The radiator is a variably polarizing undulator of APPLE-II type, where APPLE stands for advanced polarizing photon light emitter [19]. This type of undulator consists of four permanent magnet rows, two above and two below midplane, which can be moved individually in 
the longitudinal direction. Shifting two diagonal rows simultaneously in the same direction changes the polarization of the generated light. The state of polarization, horizontal, elliptical or vertical, is defined by the longitudinal shift. If the same rows are moved in opposite directions linearly polarized light is produced, where the inclination angle with respect to the midplane depends on the row shift. APPLE-II type undulators are used in many third generation synchrotron radiation facilities, because they deliver the highest horizontal on-axis fields among all planar elliptical devices. The radiator has 30 periods with a period length of $56 \mathrm{~mm}$. At a minimum gap of $15.2 \mathrm{~mm}$ the maximum $K$ parameters for horizontal, circular, and vertical polarization are 4.20, 3.44, and 2.98, respectively. The dispersive section between undulators (see Figs. 1 and 2) is built from four electromagnets where the magnet centers are separated by $400 \mathrm{~mm}$. The length of each magnet is $120 \mathrm{~mm}$. The magnets are powered in a way to produce a symmetric displacement of the electron trajectory. Separation of the electron trajectory from the common axis of both undulators in the chicane allows a laser beamstop to be placed inside. The beamstop prevents flooding of the diagnostics after the radiator with the strong seed laser pulse. The undulator section is followed by a $15^{\circ}$ bending magnet separating the electron beam from the photon beam. The electrons are passed to the beam dump. THz pulses from the dump, as emitted by the short bunch, are used to verify the bunch compression.

\section{Diagnostics}

The main diagnostics used throughout the accelerator are current transformers and fluorescent screens with YAG crystals. The transverse overlap is secured by observing UV laser and electron bunch fluorescence on the YAG screens positioned before and after the modulator and overlapping them by controlling the position of the UV beam and electron bunches. Because of the long focal length $(7 \mathrm{~m})$ with which the seed laser pulse is focused, the UV beam has the approximately same small size on both screens while achieving focus inside the modulator. The electron beam size is approximately FWHM 2.5 and $1.2 \mathrm{~mm}$ and the UV beam size is 2.5 and $1.5 \mathrm{~mm}$, before and after the modulator, respectively. The resolution of the two screens is $47 \mu \mathrm{m} / \mathrm{pxl}$ and $54 \mu \mathrm{m} / \mathrm{pxl}$. The integral of a current transformer signal before the modulator undulator can be calibrated to a Faraday cup (at the dump) and thus provides an adequate charge reading.

The photon beam after the radiator is collected by a gold coated on-axis spherical mirror at $45^{\circ}$ angle of incidence. The entrance slit of a Seya-Namioka monochromator (2400 l/mm grating) is placed at the vertical focus of the resulting astigmatic beam. This narrow line focus ( $\sim 200 \mu \mathrm{m}$ wide) allows us to open the entrance slit to the spectrometer to avoid cutting photons due to pointing variations in the electron beam. Pointing variations will instead appear as a small shift in wavelength because of a displacement of the source point. The spectrometer resolution is $0.28 \mathrm{~nm}$ with a $0.038 \mathrm{~nm} /$ pixel dispersion. The signal is recorded on a liquid nitrogen cooled CCD array $(330 \times 1100$ pixels $)$.

The precise temporal overlap between the electron bunch and the laser pulse is achieved by an electro-optical spectral decoding (EOSD) system [20] providing a sub-ps measure on the relative arrival times of the two pulses. Stability of the longitudinal overlap between the seed laser pulse and the electron bunch is improved by adding a feedback loop from the EOSD system to the seed laser delay stage. This ensures generation of higher harmonics in every shot [21]. Compression of the electron bunch is achieved by adjusting magnets and $\mathrm{rf}$ phases of the fields inside the linear accelerator for maximum compression to which fine adjustments are done while monitoring the width of the signal on the EOSD system. The measured bunch length is $1 \mathrm{ps}$ (FWHM).

\section{RESULTS AND DISCUSSION}

\section{A. Harmonics}

CHG was observed in the linear mode of the radiator at all harmonics of the seed laser up to 6th $(44 \mathrm{~nm})$ at bunch charges of $25 \mathrm{pC}$ and $2 \mathrm{~Hz}$ repetition rate. After switching to the circular mode by shifting two of the magnetic rows of the radiator, a circular coherent emission up to the 4th harmonic $(66 \mathrm{~nm})$ has also been recorded.

Single shot spectra taken at the 2nd harmonic $(131 \mathrm{~nm})$ in circular mode of the radiator are depicted in Fig. 3 revealing that a coherent signal occurs at every shot but

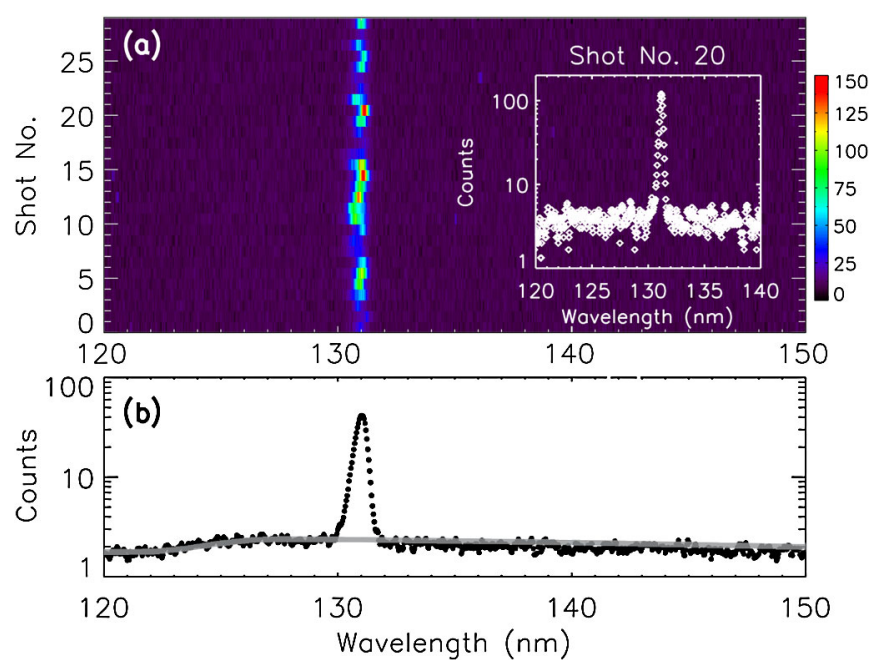

FIG. 3. A series of single shots taken at the 2nd harmonic at $131 \mathrm{~nm}$ (a) and the time averaged intensity over these 30 shots (b). A single shot spectrum is depicted in the inset. The calculated incoherent undulator harmonic was fitted for the pulse energy calibration [gray line in (b)]. These measurements were done before stability improvements on laser-electron overlap. 
with fluctuating intensity. The appearing shot-to-shot variation in wavelength arises from the pointing stability of the radiator beam passing the monochromator at different positions through the large entrance slit of $1 \mathrm{~mm}$. The highest coherent signal compared to the incoherent background was a factor of 78 at $25 \mathrm{pC}$ bunch charge.

All measured coherent peaks at any harmonics exclusively occur due to radiation from the radiator while coherent emission from the dipole magnet (dump) is negligible. This fact is proven by the complete disappearance of the coherent signals in case of detuning the radiator (not shown). Changing the resonance of the radiator to the 4th harmonic of the laser, a corresponding trace of single shots could be recorded in the planar mode of the radiator as well, as shown in Fig. 4. Again, the signal compared to the incoherent light is fluctuating shot to shot but is typically smaller than 10. A pulse energy of $3.3 \mathrm{pJ}$ could be estimated by calibrating each shot by the incoherent light appearing as the broad spectral feature beneath the narrow CHG peak. Changing the radiator shift parameter (see Fig. 1) to circular emission, $\mathrm{CHG}$ at the 4th harmonic $(66 \mathrm{~nm})$ of the same order of magnitude is observed (see Fig. 5). According to our calculations using the measured real field map in this case the degree of circular polarization yields $S 3 / S 0=0.89$ at $66 \mathrm{~nm}$. Here $\mathrm{S} 3$ and $\mathrm{S} 0$ are the Stokes vectors. The obvious difference to the ideal case $S 3 / S 0=1.0$ is due to the large angular acceptance $(\geq 1 / \gamma)$ of the detection setup and due to a slight misalignment of the magnetic row phasing. The polarization degree of coherent light is assumed to be equally high as in the incoherent case as it is based on the circular trajectory of the electrons only. The polarization degree of spontaneous circular VUV light from the same type of undulator
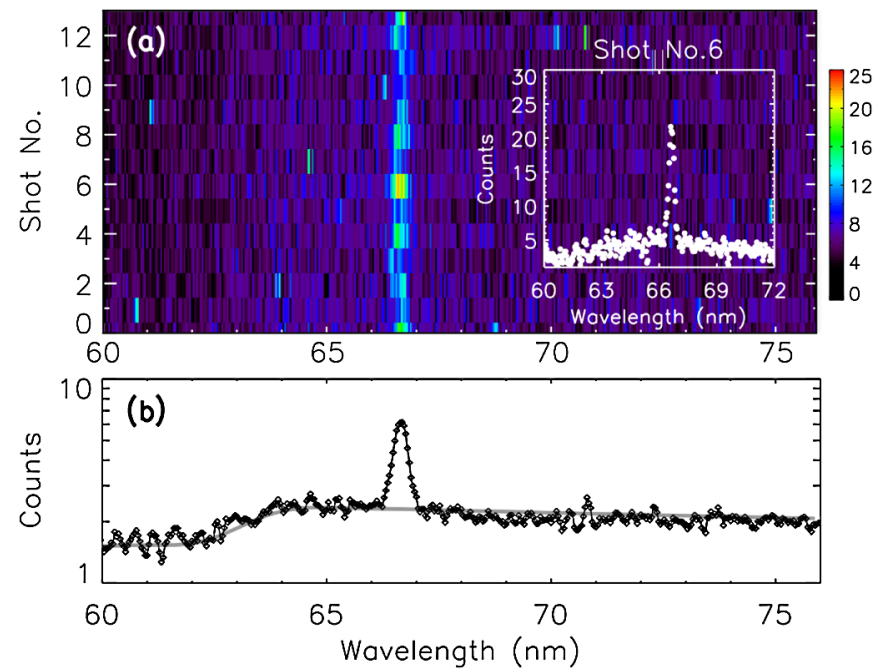

FIG. 4. A series of color coded single shots (a) and the average of them (b) recorded in the linear mode of the radiator at $66 \mathrm{~nm}$. A single shot spectrum is plotted in the inset. The calculated incoherent undulator harmonics (light gray line) is fitted for the pulse energy calibration.

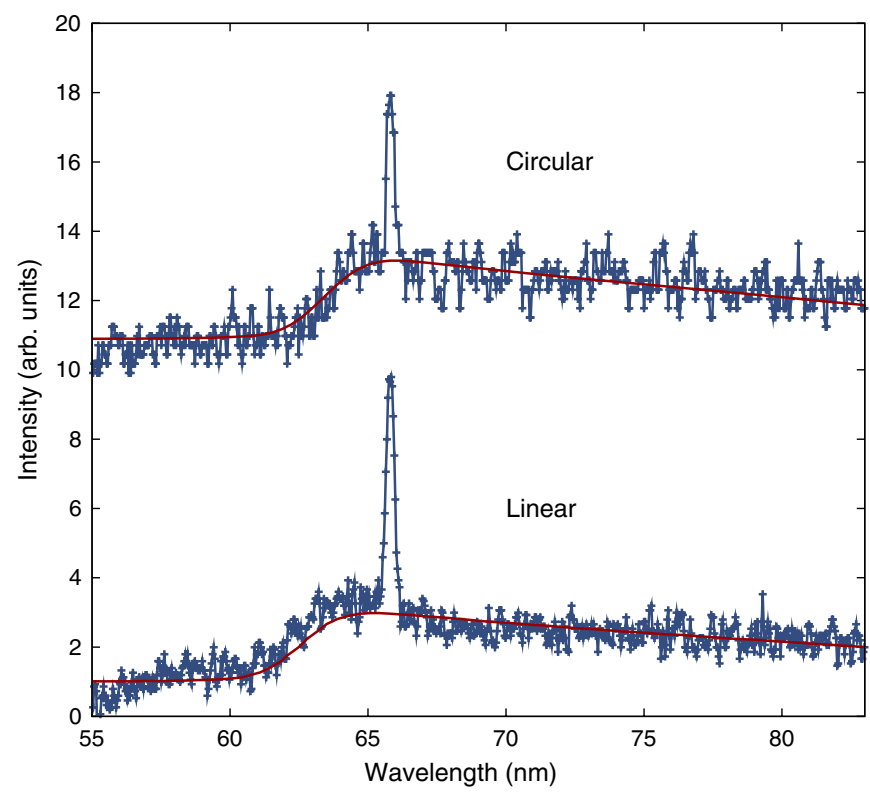

FIG. 5. Coherent 4th harmonic in circular and linear polarization. The full lines indicate the expected incoherent undulator harmonic according to SPECTRA simulations. The coherent gain is of the same order of magnitude in both cases and its difference is within the margins of overall stability fluctuations of the system.

(UE56) has been carefully measured and compared to undulator theory at the BESSY II storage ring [22]. The agreement is within the measurement accuracy of 3\%.

Figure 6 shows coherent harmonics on wavelengths corresponding to the fourth and sixth harmonic of the seed pulse. The radiator is set to $131 \mathrm{~nm}$ so that these two harmonics are second and third harmonic of the radiator. The $44 \mathrm{~nm}$ signal is relatively weak. Harmonics with wavelengths of 88 and $52 \mathrm{~nm}$ in linear polarization were also produced but are not presented in this work.

The measured linewidth of the coherent photon pulses (Fig. 7) is broadened due to the resolution of the spectrometer. Taking the broadening into account and assuming transform limited Gaussian pulses, the pulse duration was

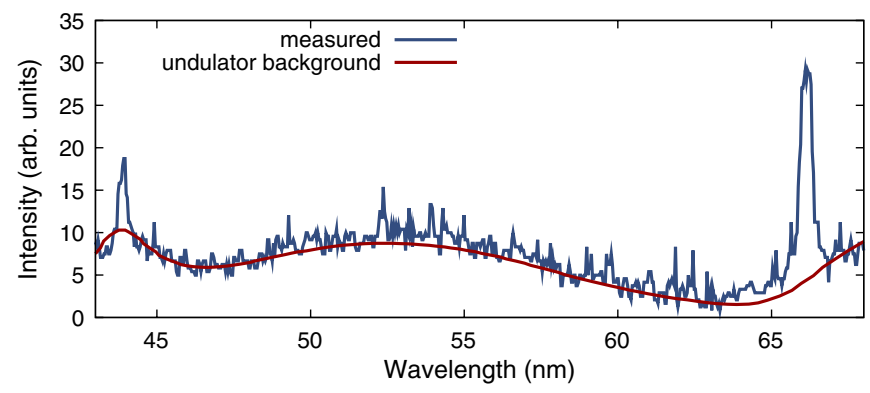

FIG. 6. Coherent fourth and sixth harmonic (66 and $44 \mathrm{~nm}$ ) in linear polarization. Incoherent undulator harmonic background indicated separately. The radiator undulator is set to the second harmonic of the modulator undulator $(131 \mathrm{~nm})$. 


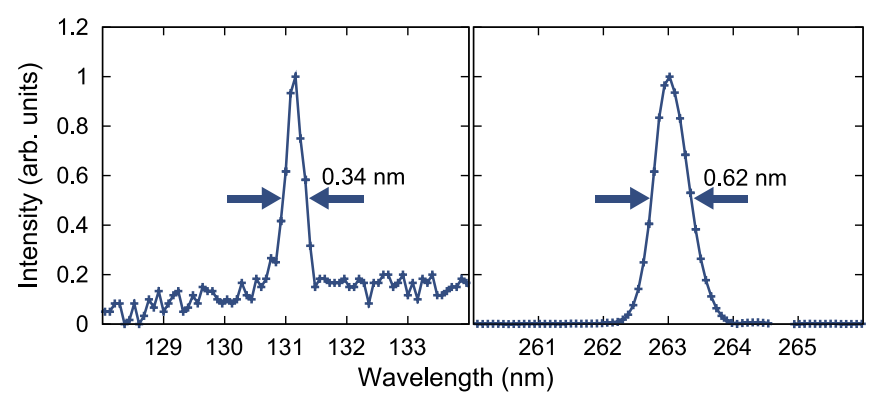

FIG. 7. Full-width half maximum linewidth of a peak measured at 131 and $263 \mathrm{~nm}$ is used to estimate the pulse length. Both spectra are normalized. The noise is less prominent on the fundamental due to higher intensity.

estimated to be 200 fs. Start-to-end simulations [23] suggested a pulse length of 150 fs (FWHM) which corresponds well to the measured values.

\section{B. Energy modulation and microbunching}

Clear evidence that the coherent signal originates exclusively from microbunching of the electron beam is given by Fig. 8. The disappearance of the signal is shown after detuning the resonance by $19 \mathrm{~nm}(0.6 \mathrm{~mm}$ gap change) which is larger than the bandwidth of the modulator's 1st harmonic. The shift is a factor of 60 larger than the measured laser bandwidth $(0.3 \mathrm{~nm}$ at $263 \mathrm{~nm})$ as depicted in the inset of Fig. 8.

The energy modulation depends on the spectral overlap of the laser beam and the spontaneous undulator radiation [24]. In the case of a narrow laser spectrum, a minimum width of $\Delta \lambda=\frac{\lambda}{N}=8.8 \mathrm{~nm}$ is expected, where $\lambda$ is the resonance wavelength and $N$ the number of undulator periods $(N=30)$. The measured resonance width is $11.2 \mathrm{~nm}$ wider than expected from the on-axis spectra in the inset of Fig. 8 (electron beam emittance ignored). However, detuning by $19 \mathrm{~nm}$, there is no spectral overlap

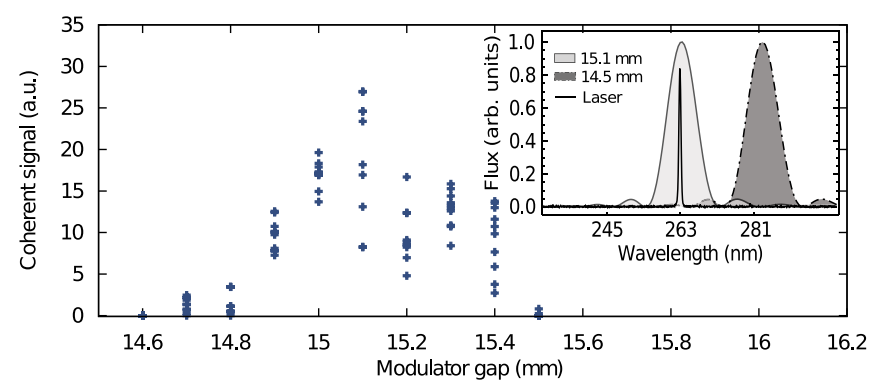

FIG. 8. Coherent signal for different gap settings of the modulator. For a given electron energy of $375 \mathrm{MeV}$ the resonance was found at $15.1 \mathrm{~mm}$ gap. No energy modulation is observed at $0.6 \mathrm{~mm}$ detuning $(19.4 \mathrm{~nm})$, which is accompanied by disappearing coherent signal. Inset: Overlap of the measured laser spectrum with calculated spontaneous spectra of the modulator for two different gaps. The seed laser is resonant with a gap of $15.1 \mathrm{~mm}$ and off-resonant with a gap of $14.5 \mathrm{~mm}$.

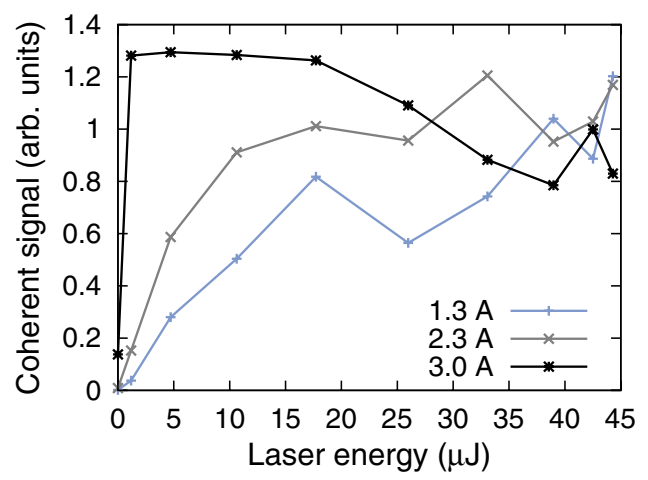

FIG. 9. Dependency of coherent signal at $133 \mathrm{~nm}$ on laser energy and chicane strength allows estimates of optimal parameters for energy modulation and compression.

between laser and undulator field anymore and bunching as well as CHG disappears as expected. The discrepancy between the expected and measured widths may be explained by suboptimal use of the length of the undulator. The central part will give the dominating modulation effect, and this gives a lower effective number of periods: $N_{\text {eff }}<N$, and thus a larger line width.

To determine optimal bunching conditions that produce strongest coherent signals, we scanned the dependence of the coherent signal at $131 \mathrm{~nm}$ relative to the strength of the chicane and the seed laser energy (Fig. 9). The signal strength was averaged over 20 consecutive shots after which the laser energy was changed. The results indicate overbunching with strong chicane and high laser energy since the coherent signal drops. The sensitivity to the laser energy sharply increases with chicane strength for small laser intensities. The driving currents through the chicane dipoles were $1.3,2.3$, and $3 \mathrm{~A}$ for which the corresponding $\mathrm{R}_{56}$ parameters were $-0.091,-0.283$, and $-0.483 \mathrm{~mm}$ (respectively, calculated from field strengths and geometry).

\section{CONCLUSION}

The MAX-lab test-FEL facility in Lund produces linearly and circularly polarized harmonics of a tripled Ti: sapphire system. The most interesting case of those, the $66 \mathrm{~nm}$ in circular polarization, with $25 \mathrm{pC}$ of electron bunch charge is estimated to give $3 \mathrm{pJ}$ in pulse energy. It is shown that microbunching is the main reason of coherent signal based on the disappearance of the signal for a detuned modulator undulator. The pulse length is estimated based on spectral width of the harmonics to be on the order of $200 \mathrm{fs}$. Further upgrades to the system are in progress to measure transverse coherence and directly characterize the polarization.

Although the original target for the MAX-lab test facility has been reached (extraction of the 5th harmonic in linear mode) and surpassed, measurements on the harmonics will continue. The main goal of the facility is investigation, preparation, and training for future FEL facilities, 
such as the possible MAX IV FEL [25]. The project will during the coming year focus on the characterization of the generated CHG pulses, tests, and investigation of diagnostics and seeding with a focus of providing a firmer platform for the MAX IV FEL development.

\section{ACKNOWLEDGMENTS}

We thank A. Gaupp and A. Meseck for helpful discussions. This work is partly funded by IRUVX-PP, an EU cofunded project under FP7 (Grant Agreement No. 211285), the European Research Council (Grant ALMA 227906), and the Swedish Research Council.

[1] M. Abo-Bakr, J. Feikes, K. Holldack, P. Kuske, W. B. Peatman, U. Schade, G. Wüstefeld, and H.-W. Hübers, Phys. Rev. Lett. 90, 094801 (2003).

[2] R.W. Schoenlein, S. Chattopadhyay, H. H. W. Chong, T. E. Glover, P. A. Heimann, C. V. Shank, A. A. Zholents, and M. S. Zolotorev, Science 287, 2237 (2000).

[3] S. Khan, K. Holldack, T. Kachel, R. Mitzner, and T. Quast, Phys. Rev. Lett. 97, 074801 (2006).

[4] T. Shintake et al., Nat. Photon. 2, 555 (2008).

[5] W. Ackermann et al., Nat. Photon. 1, 336 (2007).

[6] P. Emma et al., Nat. Photon. 4, 641 (2010).

[7] G. Lambert et al., Nature Phys. 4, 296 (2008).

[8] A. Azima et al., in Proceedings of IPAC, Kyoto, Japan (2010), TUPE009, http://accelconf.web.cern.ch/ AccelConf/IPAC10/papers/tupe009.pdf.

[9] J. Bahrdt, B. Faatz, R. Treusch, V. Miltchev, and R. Reininger, in Proceedings of FEL, Berlin, Germany (2006), MOPPH050, http://accelconf.web.cern.ch/ AccelConf/f06/PAPERS/MOPPH050.PDF.

[10] L.-H. Yu, Phys. Rev. A 44, 5178 (1991).

[11] L.-H. Yu et al., Science 289, 932 (2000).
[12] X. J. Wang, Y. Shen, T. Watanabe, J. B. Murphy, J. Rose, and T. Tsang, in Proceedings of FEL, Berlin, Germany (2006), MOAAU05, http://accelconf.web.cern.ch/ AccelConf/f06/PAPERS/MOAAU05.PDF.

[13] A. Doyuran et al., Phys. Rev. ST Accel. Beams 7, 50701 (2004).

[14] D. Xiang et al., Phys. Rev. Lett. 105, 114801 (2010).

[15] Sincrotrone Trieste, The FERMI Conceptual Design Technical Report (2007), http://www.elettra.trieste.it/ FERMI/index.php?n=Main.CDRdocument.

[16] A. Andersson, M. Eriksson, S. Werin, S. Biedron, and H. Freund, Nucl. Instrum. Methods 445, 413 (2000).

[17] B. Anderberg, Å. Andersson, M. Demirkan, M. Eriksson, L. Malmgren, and S. Werin, Nucl. Instrum. Methods 491, 307 (2002).

[18] S. Thorin, N. Cutic, F. Lindau, S. Werin, and F. Curbis, Nucl. Instrum. Methods 606, 291 (2009).

[19] S. Sasaki, Nucl. Instrum. Methods Phys. Res., Sect. A 347, 83 (1994).

[20] N. Cutic, C. Erny, F. Lindau, and S. Werin, Nucl. Instrum. Methods 626, 16 (2011).

[21] N. Cutic, F. Lindau, S. Werin, and E. Mansten, in Proceedings of FEL, Malmö, Sweden (2010), THOA4, http://accelconf.web.cern.ch/AccelConf/FEL2010/papers/ thoa4.pdf.

[22] M. Weiss et al., Nucl. Instrum. Methods 467, 449 (2001).

[23] S. Thorin, M. Brandin, S. Werin, K. Goldammer, and J. Bahrdt, Phys. Rev. ST Accel. Beams 10, 110701 (2007).

[24] A. A. Zholents and K. Holldack, in Proceedings of FEL, Berlin, Germany (2006), THPPH059, http://accelconf .web.cern.ch/AccelConf/f06/PAPERS/THPPH059.PDF.

[25] N. Cutic, M. Eriksson, F. Lindau, S. Thorin, and S. Werin, in Proceedings of FEL, Malmö, Sweden (2010), WEPA11, http://accelconf.web.cern.ch/AccelConf/FEL2010/papers/ wepa11.pdf. 